Rev. Elev. Méd. vét. Pays trop., 1972, 25 (4) : 531-538

\title{
Parasitisme et mortalité chez les veaux malgaches Influence du déparasitage sur la composition des troupeaux
}

\author{
par P. DAYNES $\left({ }^{*}\right)$ et A. BOUCHET (*)
}

\begin{abstract}
RESUME
Les auteurs, après avoir rappelé les résultats généraux obtenus par enquêtes à Madagascar, fơnt état d'une étude effectuée dans une région délimitée.

Les contrôles coproscopiques des jeunes veaux révèlent un parasitisme important dominé par l'ascaridose qui se complique d'une strongyloidose. Ces deux parasitoses sont remplacées vers le 5 e mois par des strongles digestifs.

Une étude de compositions de troupeaux montre une mortalité atteignant 40 p. 100 des veaux de 0 à 6 mois, que les auteurs rattachent en partie au parasitisme élevé. Cette étude montre également qu'un déparasitage systématique a pu diminuer cette mortalité de $25 \mathrm{p} .100$ et les auteurs expliquent pourquoi on peut espérer faire encore mieux.
\end{abstract}

\section{INTRODUCTION}

Le parasitisme a la réputation, solide et justifiée, d'être plus important, plus répandu, plus lourd de conséquences en pays chaud et humide qu'ailleurs.

A Madagascar, il a été rendu responsable do nombreuses pertes économiques dont les plus sensibles sont les mortalités sévissant surtout chez les veaux et contribuant à la structure actuelle du troupeau.

Il est donc évident que le parasitisme helminthique doit être étudié sous tous les angles possibles.

Dans une précédente communication (1) nous faisions état des résultats d'ensemble obtenus à Madagascar dans diverses régions de l'Ile, régions représentant autant de zones climatiques différentes.

(*) I.E.M.V.T., Laboratoire Central de l'Elevage, B.P. $\square^{\circ}$ 862, Tananarive.
Les enquêtes réalisées par numérations coproscopiques sur des animaux d'élevage semiextensif (parcage le soir) montraient que plus de 90 p. 100 des animaux sont parasités à l'âge de 1 à 3 mois.

L'infestation par ascaris, très fréquente chez les plus jeunes animaux diminue ensuite dès que ceux-ci atteignent l'âge de 2 à 3 mois. L'infestation par strongyloîdes présente une courbe identique à celle de l'ascaridose.

Quand ces deux parasitismes diminuent en fréquence, apparaît alors l'infestation par les strongles digestifs, infestation qui va croître en fréquence jusqu'à atteindre plus de 70 p. 100 des animaux âgés de 3 à 7 mois.

Il apparaît que l'allure générale des courbes traduisant les résultats obtenus est sensiblement la même quelle que soit la région considérée et le climat en cause, la seule différence étant un léger décalage sur l'axe des ordonnées. 
Il nous a paru intéressant d'approfondir ces résultats par une étude plus ponctuelle portant sur une seule région.

Nous nous proposons done d'apporter ici une contribution à la connaissance du parasitisme chez les jeunes et à la mortalité qui peut en découler en nous appuyant sur les résultats d'enquêtes helminthologiques et sur des études statistiques de compositions de troupeaux. Les unes et les autres ont été effectuées dans la Province de Majunga aux environs de Tsaramandroso (sous-préfecture d'Ambato-Boeni), de Mampikony et de Port-Bergé.

Nous essayerons ensuite de dégager les avantages d'un déparasitage systématique à la lueur de ces études de compositions de troupeaux dont certaines ont été faites avant toute intervention de déparasitage et d'autres après que l'on ait mis en place et maintenu une opération systématique de déparasitage bisannuel des veaux.

\section{ENQUETES HELMINTHOLOGIQUES}

La région étudiée est située au nord-ouest de Madagascar, c'est une plaine côtière de basse altitude caractérisée par un climat tropical humide, avec une saison sèche de sept mois. La température moyenne est de $35^{\circ}$ pour le mois le plus chaud et $17,5^{\circ}$ pour le mois le plus froid, la pluviométrie est de $1.600 \mathrm{~mm}$ et essentiellement répartie de décembre à février.

Les résultats des enquêtes helminthologiques portent sur 2.718 animaux. Elles intéressent surtout des veaux, animaux âgés de moins de 1 an, et quelques animaux de plus de 1 an, mais de moins de 18 mois. Tous les animaux examinés sont parqués chaque soir.

Ces enquêtes ont été effectuées pour moitié en milieu de saison des pluies et pour moitié en milieu de saison sèche (janvier et juillet).

Les méthodes employées sont la numérotation selon MacMASTER en solution de sulfate de zinc à 33 p. 100 et la sédimentation lente.

La fréquence du parasitisme est étudiée en fonction de l'âge des animaux répartis en 7 groupes:

"1. Animaux de 0 à 15 jours.

2. Animaux de 15 jours à 1 mois.
3. Animaux de 1 à 2 mois.

4. Animaux de 2 à 3 mois.

5. Animaux de 3 à 7 mois.

6. Animaux de 7 à 12 mois.

7. Animaux de 12 à 18 mois.

Nous ne retiendrons que l'infestation par les Nématodes. L'infestation par les amphistomes n'a pratiquement pas d'incidence sur l'élevage. L'infestation par les Moniezia bien qu'étant parfois décelable chez les plus jeunes est encore faible en général chez les veaux. L'infestation par Fasciola gigantica n'existe pas actuellement dans la région considérée (on la recherche régulièrement).

\section{A. RESULTATS GLOBAUX}

\section{Nature de l'infestation parasitaire}

Les parasites gastro-intestinaux mis en évidence sont :

- Neoascaris vitulorum (Goeze 1782).

- Strongyloïdes papillosus (Weld 1856).

- Oesophagostomum radiatum (Rudolphi 1803).

- Cooperia pectinata (Ransom 1907).

- Bunostomum phlebotomum (Railliet 1900).

- Haemonchus contortus (Rudolphi 1803).

- Trichostrongylus (Leoss 1905).

- Moniezia sp. (Blanchard 1891).

- Paramphistomes divers.

- Coccidies.

\section{Taux d'infestation}

Le pourcentage d'animaux à coproscopie positive par rapport aux animaux examinés apparaît par groupe d'âges dans le tableau $n^{\circ} \mathbf{I}$ et au graphique $\mathrm{n}^{\mathrm{o}} 1$.

Les chiffres obtenus par la coproscopie sont entachés d'une marge d'erreur par défaut, la méthode pouvant donner des réponses faussement négatives. Ils montrent l'importance du parasitisme chez les animaux les plus jeunes. Ceux concernant les animaux de moins de 1 mois sont très au-dessous de la réalité puisque les animaux déjà infestés d'ascaris à la naissance n'éliminent cependant pas encore d'œufs d'ascaris dans leurs excréments. 
TABLEAU $\mathrm{H}^{\circ} \mathrm{I}$

\begin{tabular}{|c|c|c|c|c|c|c|c|c|c|}
\hline Age des animaux & & $\begin{array}{l}0 \text { a } 15 \\
\text { jours }\end{array}$ & $\begin{array}{l}2 \\
15 \text { jours } \\
\text { à } 1 \text { mois }\end{array}$ & $\begin{array}{l}3 \\
1 \text { à } 2 \\
\text { mois }\end{array}$ & $\begin{array}{l}4 \\
2 \text { à } 3 \\
\text { mois }\end{array}$ & $\begin{array}{l}5 \\
3 \text { à } 7 \\
\text { mois }\end{array}$ & $\begin{array}{c}7 \text { à } 12 \\
\text { mo is }\end{array}$ & $\begin{array}{c}12 \text { à } 18 \\
\text { mois }\end{array}$ & $\begin{array}{c}\text { B } \\
\text { Totaux }\end{array}$ \\
\hline $\begin{array}{l}\text { Nombre d'animaux } \\
\text { examinés }\end{array}$ & A & 26 & 82 & 189 & $30]$ & 1.059 & 716 & 345 & 2.718 \\
\hline $\begin{array}{l}\text { Nombre d'animaux } \\
\text { à coproscopie } \\
\text { positıve }\end{array}$ & B & 5 & 54 & 155 & 258 & 901 & 454 & 179 & 2.002 \\
\hline Pourcentage & c & 19,2 & 65,9 & 82 & 85,7 & B5 & 63,4 & 50,7 & 73,7 \\
\hline
\end{tabular}

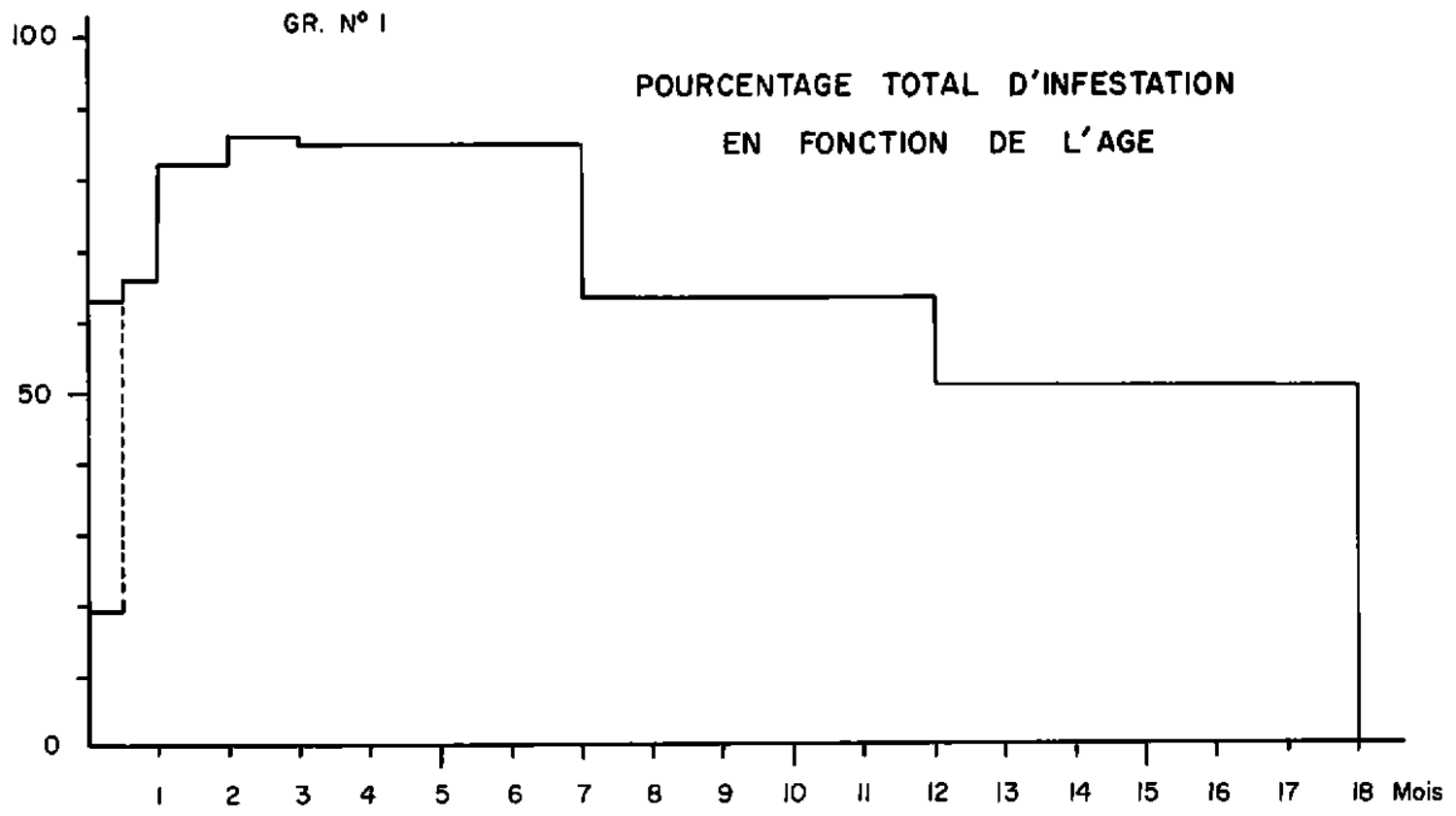

\section{B. RESULTATS PAR HELMINTHE EN CAUSE}

Les résultats retenus sont ceux correspondant aux ascaris, aux strongyloïdes et aux strongles digestifs. Dans cette dernière catégorie nous incluons indifféremment les Hoemonchus, les cesophagostomes, les Cooperia, les trichostrongles et les bunostomes.

Nous avons calculé le pourcentage d'animaux à coproscopie positive pour tel ou tel parasite, d'une part par rapport aux animaux examinés, d'autre part par rapport aux animaux reconnus parasités. Ce dernier pourcentage donne une idée de l'importance particulière de tel helminthe dans le parasitisme général.
La numération des œufs d'helminthes en cellules de MacMASTER permet d'obtenir le nombre moyen d'oufs par gramme d'excrément, ce qui donne une idée de l'intensité du parasitisme.

\section{Infestation par ascaris}

Les pourcentages d'animaux à coproscopie positive pour ascaris apparaissent dans le tableau $n^{\circ}$ II ci-dessous et au graphique $n^{*} 2$.

La coproscopie fait apparaître un maximum d'animaux parasités à l'âge de 1 à 2 mois.

Cependant, du fait de l'infestation transplacentaire du veau, il ne peut $y$ avoir moins d'animaux parasités à la naissance qu'à 2 mois. 
TABLEAU $\mathrm{N}^{\bullet}$ II

\begin{tabular}{|c|c|c|c|c|c|c|c|c|c|c|}
\hline & & Age des animaux & $\begin{array}{l}1 \\
0 \stackrel{1}{a} 15 \\
\text { jours }\end{array}$ & $\begin{array}{l}{ }^{2} \\
15 \text { jours } \\
\text { à } 1 \text { mois }\end{array}$ & $\begin{array}{l}3 \stackrel{3}{1} 2 \\
\text { mois } 2\end{array}$ & $\begin{array}{l}4 \\
2 \text { à } 3 \\
\text { mois }\end{array}$ & $\begin{array}{l}5 \\
3 \text { à } 7 \\
\text { mois }\end{array}$ & $\begin{array}{l}6 \\
7 \underset{\text { à }}{12} \\
\text { mois }\end{array}$ & $\begin{array}{l}12^{7} \text { à } 18 \\
\text { mois }\end{array}$ & $\begin{array}{c}8 \\
\text { Totaux }\end{array}$ \\
\hline \multirow{3}{*}{ 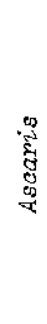 } & A & $\begin{array}{l}\text { Nombre d'animaux à } \\
\text { coproscopie positive }\end{array}$ & (4) & (32) & 116 & 160 & 209 & 12 & 0 & 533 \\
\hline & B & $\begin{array}{l}\text { Pourcentage par rapport } \\
\text { au total des animaux } \\
\text { examinês }\end{array}$ & $(15,4)$ & (39) & 61,4 & 53,2 & 19,7 & 1,7 & - & 19,6 \\
\hline & $\mathrm{C}$ & $\begin{array}{l}\text { Pourcentage par rapport } \\
\text { dux animaux reconnus } \\
\text { parasitês par la co- } \\
\text { proscopie }\end{array}$ & $(80)$ & $(59,3)$ & 74,8 & 62,0 & 23,2 & 2,6 & - & 26,6 \\
\hline \multirow{3}{*}{ 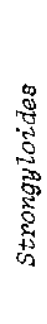 } & $\mathrm{A}_{2}$ & $\begin{array}{l}\text { Nombre d'animaux à } \\
\text { coproscopie positive }\end{array}$ & 1 & 26 & 68 & 77 & 50 & 5 & 0 & 235 \\
\hline & $\mathrm{B}_{2}$ & $\begin{array}{l}\text { Pourcentage par rapport } \\
\text { au total des animaux } \\
\text { examinés }\end{array}$ & 3,8 & 31,7 & 35,9 & 25,6 & 5,5 & 0,7 & - & 8,6 \\
\hline & $\mathrm{c}_{2}$ & $\begin{array}{l}\text { Pourcentage par rapport } \\
\text { aux animaux reconnus } \\
\text { parasitês par la } \\
\text { coproscopie }\end{array}$ & 20 & 48,1 & 43,9 & 29,8 & 6,4 & 1,1 & - & 11,7 \\
\hline \multirow{3}{*}{ 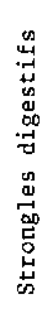 } & $\mathrm{A}_{3}$ & $\begin{array}{l}\text { Nombre d'animaux } \dot{a} \\
\text { coproscopie positive }\end{array}$ & 1 & 10 & 56 & 146 & 772 & 411 & 149 & 1.545 \\
\hline & $\mathrm{B}_{3}$ & $\begin{array}{l}\text { Pourcentage par rapport } \\
\text { au total des animaux } \\
\text { examinés }\end{array}$ & 3,8 & 12,2 & 29,6 & 48,5 & 72,9 & 57,4 & 43,2 & 56,8 \\
\hline & $\mathrm{C}_{3}$ & $\begin{array}{l}\text { Pourcentage par rapport } \\
\text { aux animaux reconnus } \\
\text { parasités par la } \\
\text { coproscopie }\end{array}$ & 20 & 18,5 & 36,1 & 56,6 & 85,7 & 90,5 & 85,1 & 77,2 \\
\hline
\end{tabular}




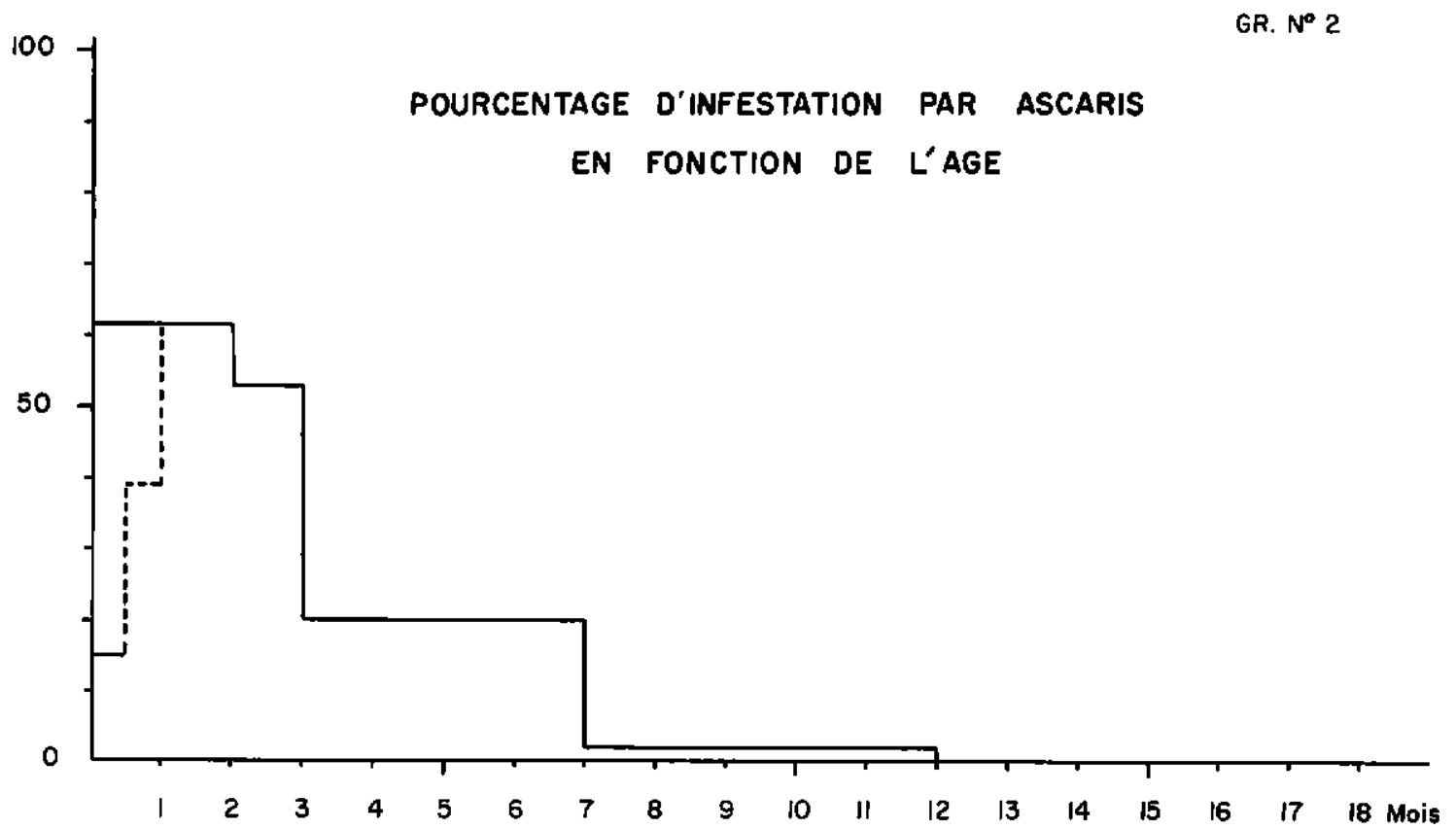

C'est pourquoi les chiffres correspondant aux animaux de 0 à 15 jours et de 15 jours à 1 mois sont entre parenthèses. Entre la naissance et 1 mois d'âge, il y a au moins 62 p. 100 des veaux qui sont parasités par ascaris, soit 2 à 4 fois plus que n'en indique la coproscopie.

La coproscopie permet donc de dire que près des $2 / 3$ des nouveau-nés sont infestés par ascaris. Cette fraction d'animaux infestés diminue avec l'âge, assez rapidement dès le $3^{+}$mois et après le $7^{e}$ mois il n'y a presque plus d'animaux infestés, ce qui est dû à la faible longévité des ascaris.
L'intensité moyenne de cette infestation est donnée par le chiffre moyen d'œufs d'ascaris par gramme d'excrément. Celui-ci atteint 8.000 chez les animaux âgés de 1 mois environ. Il descend rapidement jusque vers l'âge de 3 mois (2 à 3.000), puis plus lentement jusque vers le $10^{\mathrm{e}}$ mois.

\section{Infestation par strongyloïdes}

Le pourcentage maximal d'animaux à coproscopie positive pour strongyloïdes est de l'ordre de 36 et se situe au $2^{\mathrm{e}}$ mois (tableau $n^{\circ}$ II et graphique $n^{*} 3$ ).

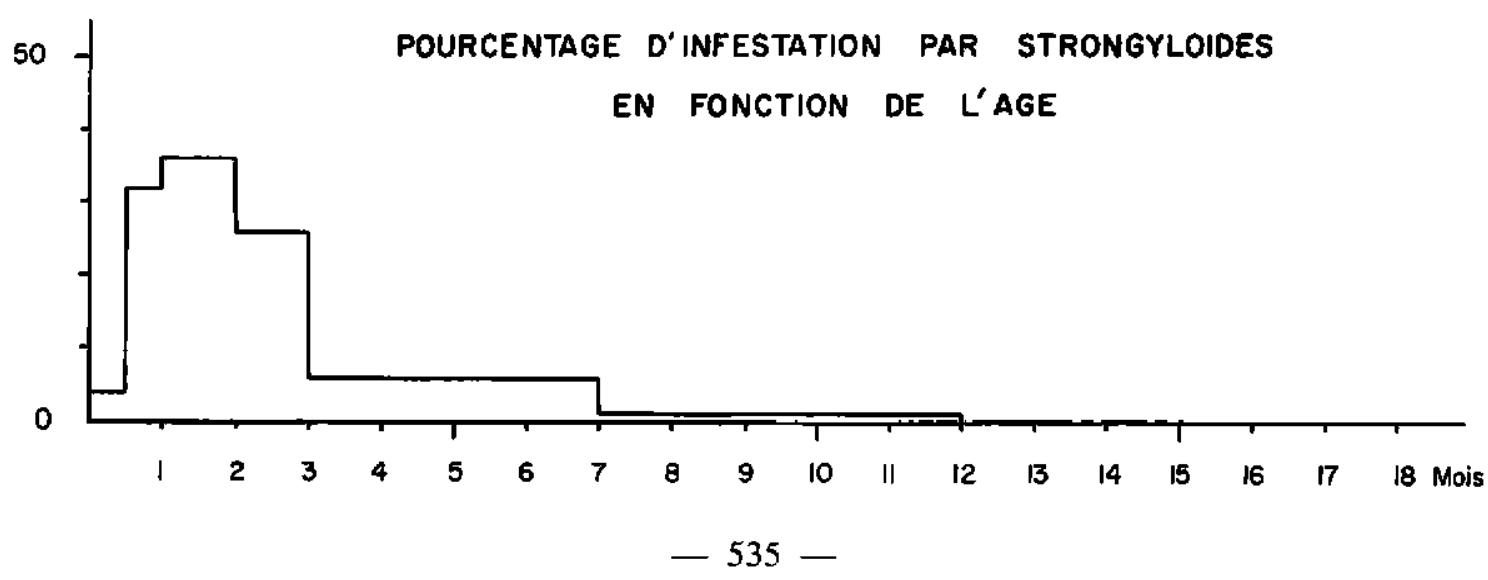


L'évolution de ce pourcentage ressemble à ce que l'on constate pour l'ascaridose. La diminution amorcée dès le $3^{\mathrm{e}}$ mois conduit à un chiffre très faible dès le $7^{\mathrm{e}}$ mois.

Le nombre moyen d'œufs par gramme d'excrèments est de l'ordre de 1.500 entre 1 et 2 mois. Il descend vers 900 à 3 mois, puis plus lentement, pour être très faible vers 10 ou 12 mois.

\section{Infestation par les strongles digestifs}

Le pourcentage d'animaux à coproscopie positive augmente avec l'âge jusque vers le $5^{\mathrm{e}}$ mois où $73 \mathrm{p} .100$ des animaux sont reconnus infestés par la coproscopie. Ce pourcentage diminue lentement mais reste supérieur à $43 \mathrm{chez}$ les animaux de plus de 12 mois.

Le nombre moyen d'cufs par gramme d'excréments passe lentement de $300 \mathrm{chez}$ les animaux de 1 à 2 mois à 800 chez les animaux de 12 mois et plus.

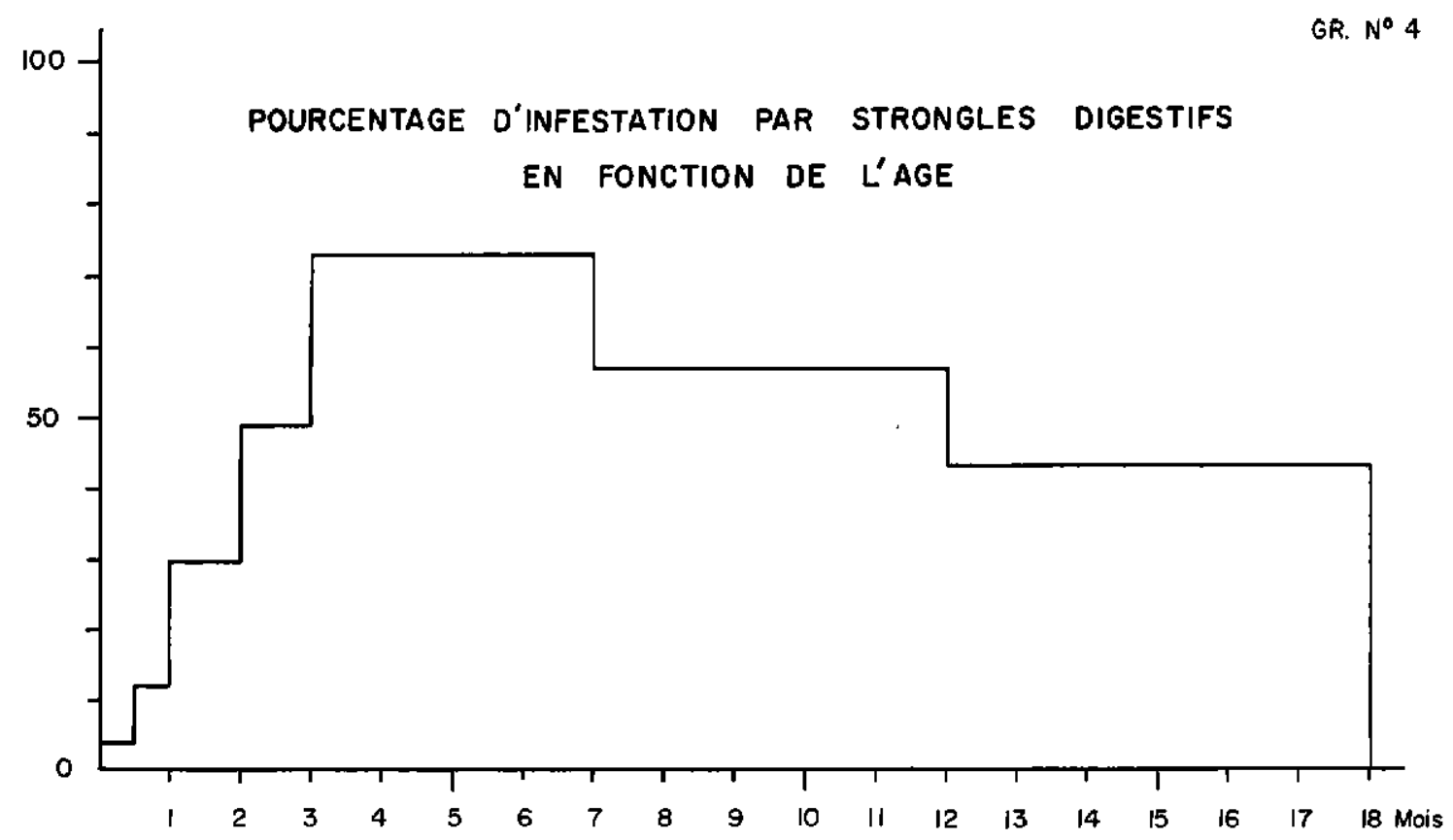

\section{DISCUSSION}

Les tableaux I et II et les graphiques correspondants montrent un taux d'infestation important chez les tout jeunes veaux de moins de 2 mois.

Le parasitisme du nouveau-né est dominé par l'ascaridose à laquelle se surajoute rapidement la strongyloïdose. Ces deux helminthoses vont décroître rapidement en fréquence mais les strongyloses digestives s'installent et entre le $2^{e}$ et le $3^{e}$ mois prennent en fréquence la place qu'occupait l'ascaridose.

Les nombres moyens d'œufs par gramme d'excréments sont également éloquents : 8.000 pour l'ascaris chez les animaux de 1 à 2 mois, 5 à 600 pour les strongles digestifs chez les animaux de 6 mois. Ces chiffres ne sont pas tellement éloignés de ceux habituellement retenus (2) comme limite de l'infestation - maladie en milieu tempéré et monoparasitisme. En milieu tropical, la malnutrition quasi permanente augmente la sensibilité au parasitisme et le pouvoir pathogène apparaît pour des degrés d'infestation inférieurs à ceux retenus pour les pays tempérés.

D'autre part les associations parasitaires, ascaridose, strongylö̈ose, strongyloses digestives, augmentent encore ce pouvoir pathogène. 


\section{COMPOSITIONS DE TROUPEAUX}

Des compositions de troupeaux ont été effectuées dans la région considérée, d'une part fin 1969, d'autre part en 1971, juillet et novembre.

Les compositions de troupeaux de 1969 portent sur 4.500 animaux et les compositions de 1971 sur 8.125 animaux.

Elles ont été faites en même temps qu'un déparasitage des veaux. On peut considérer que l'ensemble des femelles, et l'ensemble des veaux étaient présents.

Le taux de naissances observé pour la région étudiée est de l'ordre de 65 p. 100 . Il correspond au rapport des naissances annuelles sur le nombre de "vaches".

LACROUTS (3) admet que les vaches sont les femelles de plus de trois ans. Nous pensons qu'il faut plutôt considérer les femelles de plus de 4 ans et ce sont celles-ci que nous appelons vaches.

A partir de nos relevés, nous avons établi le pourcentage de veaux présents lors de nos enquêtes par rapport aux vaches présentes également. Ce pourcentage était de 39 en 1969. Il était de 45,6 en 1971.

Si l'on compare ces pourcentages au taux de naissance moyen de 65 p. 100 , on en déduit une mortalité des nouveau-nés de 39,9 p. 100 (1969) ou de 29,8 p. 100 (1971), mortalité ayant sévi entre la naissance des animaux et le moment des observations.

L'âge moyen des veaux de moins d'un an lors des observations peut être considéré comme étant 6 mois; les naissances ne sont pas également réparties au cours de l'année mais les observations ayant été faites en plusieurs mois (septembre à décembre en 1969), (juin, juillet et novembre, décembre en 1971), l'approximation est valable pour notre propos.

On peut en conclure que 29,8 p. 100 ou 39,9 p. 100 des veaux sont morts entre leur naissance et l'âge de 6 mois, suivant que l'on a déparasité ou non.

\section{CONCLUSIONS}

Il est intéressant de rapprocher les chiffres obtenus par l'étude des compositions de trou- peaux des résultats obtenus par la coproscopie des veaux.

Les premiers indiquent que 30 à 40 p. 100 des veaux nés ont disparu avant d'atteindre l'âge de 6 mois. Or, la simple observation des graphiques $1,2,3$ et 4 montre un taux élevé d'animaux infestés chez les plus jeunes et particulièrement chez les animaux de moins de 6 mois.

Même si le parasitisme interne ne peut être tenu pour le seul responsable de la mortalité des veaux, il y contribue certainement pour une grande part. La malnutrition frappe tous les veaux et les plus parasités y sont évidemment plus sensibles que les autres.

L'étude des graphiques montre également la nécessité d'effectuer précocement les traitements anthelminthiques éventuels.

S'il apparaît que l'on a intérêt à lutter contre la strongylö̈dose dès le premier mois de vie, il est encore plus important de traiter contre l'ascaridose dès la naissance des animaux.

Ultérieurement il faut traiter contre les strongyloses digestives. Si le pourcentage d'animaux infestés semble régresser légèrement nous avons vu que, par contre, l'intensité de leur infestation augmente. Ce déparasitage se situera judicieusement à la période du sevrage qui constitue une transition difficile pour le jeune animal.

Les chiffres obtenus par l'étude des compositions de troupeaux sont également instructifs à un titre différent. Ils montrent l'impact que peut avoir sur l'élevage une entreprise de déparasitage systématique des jeunes.

Les compositions de troupeaux de 1969 portaient sur des troupeaux non déparasités. Celles de 1971 portaient sur des troupeaux où un déparasitage des veaux a été effectué pratiquement 2 fois par an depuis la fin 1969.

Plus précisément les veaux des troupeaux vus en jujllet 1971 avaient été déparasités en février 1971, pour ceux qui étaient déjà nés, et les veaux des troupeaux vus en novembre 1971 avaient été déparasités en juillet et même en février pour les plus vieux.

Les chiffres semblent traduire de façon objective un effet favorable du déparasitage, 
effet perçu par ailleurs et signalé avec insistance par les paysans.

En effet le pourcentage de veaux par rapport aux vaches augmente entre 1969 et 1971 de façon sensible $(45,6$ au lieu de 39$)$ et la mortalité des veaux marque une régression de $25 \mathrm{p}$. $100(29,8$ contre 39,9$)$.

Il est bien évident qu'il convient d'être prudent dans l'interprétation des chiffres relevés, mais on ne peut manquer de noter l'influence favorable d'un déparasitage dont on sait cependant qu'il n'a pu être réalisé dans les meilleures conditions; ce déparasitage, en effet, a été réalisé en deux passages d'équipes au cours de l'année.

Une opération de déparasitage mieux conduite ne peut qu'aboutir à des résultats meilleurs encore. Lors de passage systématique d'équipes de déparasitage beaucoup d'animaux sont déjà morts, la plupart ne sont déjà plus des nouveau-nés. Or, la simple observation des graphiques de parasitisme montre l'obligation de traiter dès la naissance comme nous l'avons précisé ci-dessus. Plus le déparasitage sera fait précocement et meilleurs seront les résultats obtenus.

\section{SUMMARY}

Parasitism and death rate in malagasy calves. Influence of parasite control on herd composition

The authors first recall the general results proceeding from different investigations in Madagascar, and then present a study done in a delimited territory.

Coproscopic controls from calves show an important parasitism with ascaris and strongylids, both beeing soon overcome by digestive strongylids.

A study of compositions of herds shows a death rate reaching $40 \mathrm{p} .100$ of calves 0 to 6 months old, partly because of high parasitism, according to the authors. This study also shows that a systematic treatment possibly reduce that death rate of $25 \mathrm{p} .100$ and the authors explain why they think it is very possible to get more better results.

\section{RESUMEN}

Parasitismo y mortalidad en los terneros de Madagascar.

Influencia de la lucha contra los parásitos en la composición de las manadas

Los autores recordan los resultados generales obtenidos durante encuestas parasitológicas en Madagascar y presentan un estudio efectuado en una región delimitada.

Las comprobaciones coproscopicas de los terneros muestran un parasitismo importante dominado por la ascaridosis que se complica por una estrongiloidosis, luego substituidas por las estrongilas digestivas. Un estudio de composición de las manadas muestra una mortalidad llegando a 40 p. 100 de los terneros de 0 à 6 meses de edad, que los autores atribuyen por parte al parasitismo elevado.

Este estudio muestra tambien que una lucha sistemática contra los parásitos pudo disminuir dicha mortalidad de 25 p. 100 y los autores explican por qué se puede esperar obtener mejores resultados.

\section{BIBLIOGRAPHIE}

1. DAYNES (P.) et BOUCHET (A.). Enquêtes helminthologiques sur les nématodoses du veau. XIXe Congrès Mondial Vétérinaire, Mexico 1528 août 1971 .

2. EUZEBY (J.). Diagnostic expérimental des helminthoses animales. Paris, Vigot Frères, 1958.
3. LACROUTS (M.), TYC (J.), BERTRAND (S.), SARNIGUET (J.). Etude des problèmes posés par l'élevage et la commercialisation du bétail et de la viande à Madagascar. Tome I et II. Paris, Ministère de la Coopération, 1962. 